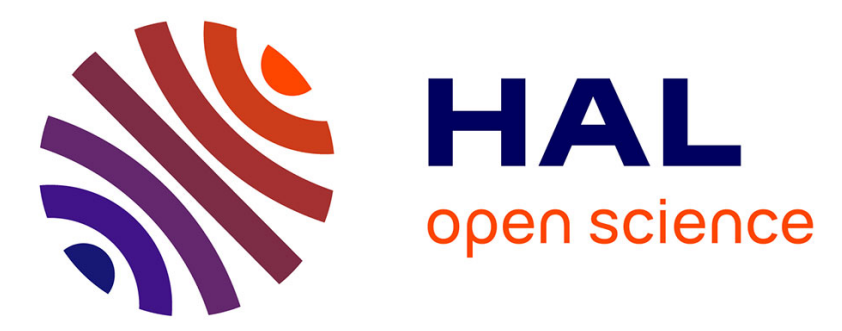

\title{
Blocking Rates in Large CDMA Networks via a Spatial Erlang Formula
}

François Baccelli, Bartlomiej Blaszczyszyn, Mohamed Kadhem Karray

\section{To cite this version:}

François Baccelli, Bartlomiej Blaszczyszyn, Mohamed Kadhem Karray. Blocking Rates in Large CDMA Networks via a Spatial Erlang Formula. [Research Report] RR-5517, INRIA. 2005, pp.19. inria-00070489

\section{HAL Id: inria-00070489 \\ https://hal.inria.fr/inria-00070489}

Submitted on 19 May 2006

HAL is a multi-disciplinary open access archive for the deposit and dissemination of scientific research documents, whether they are published or not. The documents may come from teaching and research institutions in France or abroad, or from public or private research centers.
L'archive ouverte pluridisciplinaire HAL, est destinée au dépôt et à la diffusion de documents scientifiques de niveau recherche, publiés ou non, émanant des établissements d'enseignement et de recherche français ou étrangers, des laboratoires publics ou privés. 


\section{Blocking Rates in Large CDMA Networks via a Spatial Erlang Formula}

François Baccelli — Bartłomiej Błaszczyszyn — Mohamed Kadhem Karray

$\mathbf{N}^{\circ} \mathbf{5 5 1 7}$

March 2005

THÈME 1 



\title{
Blocking Rates in Large CDMA Networks via a Spatial Erlang Formula
}

\author{
François Baccelli*, Bartłomiej Błaszczyszyn ${ }^{\dagger}$, Mohamed Kadhem Karray ${ }^{\ddagger}$ \\ Thème 1 - Réseaux et systèmes \\ Projet TREC
}

Rapport de recherche $\mathrm{n}^{\circ} 5517$ - March 2005 - 19 pages

\begin{abstract}
This paper builds upon the scalable admission control schemes for CDMA networks developed in $[2,1]$. These schemes are based on an exact representation of the geometry of both the downlink and the uplink channels and ensure that the associated power allocation problems have solutions under constraints on the maximal power of each station/user. These schemes are decentralized in that they can be implemented in such a way that each base station only has to consider the load brought by its own users to decide on admission. By load we mean here some function of the configuration of the users and of their bit rates that is described in the paper. When implemented in each base station, such schemes ensure the global feasibility of the power allocation even in a very large (infinite number of cells) network. The estimation of the capacity of large CDMA networks controlled by such schemes was made in these references. In certain cases, for example for a Poisson pattern of mobiles in an hexagonal network of base stations, this approach gives explicit formulas for the infeasibility probability, defined as the fraction of cells where the population of users cannot be entirely admitted by the base station. In the present paper we show that the notion of infeasibility probability is closely related to the notion of blocking probability, defined as the fraction of users that are rejected by the admission control policy in the long run, a notion of central practical importance within this setting. The relation between these two notions is not bound to our particular admission control schemes, but is of more general nature, and in a simplified scenario it can be identified with the well-known Erlang loss formula. We prove this relation using a general spatial birth-and-death process, where customer locations are represented by a spatial point process that evolves over time as users arrive or depart. This allows our model to include the exact representation of the geometry of inter-cell and intra-cell interferences, which play an essential role in the load indicators used in these cellular network admission control schemes.
\end{abstract}

Key-words: Processus stochastique/ Théorie de files d'attente, CDMA, contrôle d'admission décentralisé, infaisabilité, probabilité de blocage, formule d'Erlang, processus de Poisson, processus spatial de naissance-et-mort, processus de Gibbs

\footnotetext{
* INRIA-ENS, 45 rue d'Ulm 75005, Paris, France, francois.baccelli@ens.fr

† INRIA-ENS, 45 rue d'Ulm 75005, Paris, France, Bartek.Blaszczyszyn@ens.fr

¥ France Télécom R\&D, 38/40 rue du Général Leclerc, 92794 Issy-Moulineaux France, mohamed.karray@rd.francetelecom.com
} 


\section{Taux de Blocage dans les grands réseaux CDMA par l'intermédiaire d'une Formule Spatiale d'Erlang}

Résumé : Cet article s'appuie sur les algorithmes de contrôle d'admission pour des réseaux CDMA développés dans $[2,1]$. Ces algorithmes sont basés sur une représentation exacte de la géométrie sur les voies montante et descendante et assurent que les problèmes associés d'allocation de puissance ont des solutions sous des contraintes sur la puissance maximale de chaque utilisateur/station. Ces algorithmes sont décentralisés car ils peuvent être implémentés de manière que chaque station de base considère seulement la charge apportée par ses propres utilisateurs pour décider de l'admission. (Par la charge nous désignons ici une certaine fonction de la configuration des utilisateurs et de leurs débits qui est décrite dans le papier.) Une fois implémentés dans chaque station de base, ils assurent la faisabilité globale de l'allocation de puissance même dans un très grand réseau (nombre infini de cellules). L'évaluation de la capacité de grands réseaux de CDMA contrôlés par ces algorithmes a été effectuée dans ces références. Dans certains cas, par exemple pour le modèle de Poisson des mobiles dans un réseau hexagonal des stations de base, cette approche donne des formules explicites pour la probabilité d'infaisabilité; c.-à-d., pour la probabilité qu'une population de Poisson d'une intensité donnée ne peut pas être entièrement admise par les stations de base implémentant le protocole de contrôle d'admission. Dans le papier nous prouvons que la notion de la probabilité d'infaisabilité est étroitement liée à la notion de probabilité de blocage; c.-à-d., à la fraction des utilisateurs qui sont rejetés par le contrôle d'admission à longue durée, une notion très importante dans la pratique dans ce contexte. La relation entre ces deux notions n'est pas liée à nos algorithmes particuliers de contrôle d'admission, mais est d'une nature plus générale; et dans un scénario simplifié, peut être identifiée avec la formule d'Erlang classique. Nous montrons cette relation en utilisant un processus de naissance-etmort spatial, où les positions des utilisateurs sont représentées par un processus ponctuel spatial qui évolue dans le temps quand les clients arrivent ou partent. Ceci nous permet de prendre en compte de manière exacte la géométrie des interférences inter-cellulaires et intra-cellulaires, qui jouent un rôle essentiel dans les indicateurs de charge utilisés dans ces algorithmes de contrôle d'admission.

Mots-clés : $\quad$ Stochastic processes/Queueing theory, CDMA, decentralized admission control, infeasibility, blocking probability, Erlang formula, Poisson process, spatial birth-and-death process, Gibbs process 


\section{Introduction}

Consider calls arriving to some infinite server queue according to a time Poisson point process with intensity $\lambda$, and suppose each call has an exponential holding time with mean $\tau$. It is well known that the number of calls in progress observed in the steady state of such a non-constrained model is a Poisson random variable $N$ with mean $\lambda \tau$. Consider now some positive integer $C$. We define the feasibility (resp. infeasibility) probability for $C$ as $\mathbf{P}(N \leq C)$ (resp. $(\mathbf{P}(N>C))$ ). In general, these probabilities have nothing to do with the dynamics of the model where calls are rejected when their number exceeds $C$. For the latter we need to describe an admission control policy which specifies how the model performs when the $C$ limit is reached. The classical loss system simply drops the calls that arrive when there are $C$ calls already in progress. In this case, we define the blocking probability as the fraction of calls that are dropped in the long run by the system. Erlang's formula (see e.g. [9]) states that the blocking probability $b$ is

$$
b=\frac{\mathbf{P}(N=C)}{\mathbf{P}(N \leq C)}=\frac{(\lambda \tau)^{C}}{C !}\left(\sum_{n=0}^{C} \frac{(\lambda \tau)^{n}}{n !}\right)^{-1} .
$$

So Formula (1.1) shows that in spite of the differences between the dynamics of the non-constrained and that of the loss system, the blocking probability can be expressed in terms of the steady state distribution of calls in progress of the non-constrained system.

As well known, Erlang published this formula in 1917, and since that time, the statistical equilibria of much more complicated loss networks have been found to coincide with the truncation of the stationary distribution of some non-constrained system to some polytope. This lead to the calculation of the associated blocking probabilities in explicit form for large classes of networks. For an exhaustive survey on loss systems, see [9]).

Classical loss models are well adapted to wired communication networks, where the spatial component of the model is typically represented by some graph of links, and where the coexistence of calls on a common link is modeled by the occupancy of a discrete number of circuits available on this link. In wireless communication, one needs to take into account the spatial characteristics of the network in a more thorough way because it is the relative location of the radio channels which determines their joint feasibility. This is especially important for Code Division Multiple Access (CDMA) and other so called interference limited systems. One of the additional difficulties then stems from the fact that the spatial component of the model is subject to changes due to the mobility of users and instantaneous changes of radio conditions.

In CDMA, a given configuration of channels with predefined bit-rates is feasible if there exist some vector of emitted powers which guarantee that the Signal-to-Interference-and-Noise-Ratio (SINR) at each receiver exceeds some threshold defined by the bit rate of the associated channel. The solution to this power allocation problem may also be constrained by further limitations on the maximum power of stations/users.

The main contribution of the paper is the connection that is established between the notion of the feasibilty probability defined in $[2,1]$ in connection with the admission control schemes alluded to in the abstract, and the notion of blocking probability, defined again as the fraction of users that are rejected by the admission control policy in its long run.

The paper is organized as follows. In Section 2 we make a short survey of the literature on performance evaluation of load control schemes for CDMA networks. In Section 3 we recall briefly the decentralized admission control schemes for CDMA networks developed in $[2,1]$. We make the connection in question and calculate the blocking probabilities (rates) in Section 4. Some numerical examples are presented in Section 5. In Appendix we give mathematical foundations for the general spatial Erlang formula that we use in the paper. 


\section{Related Work}

There is a rich literature on the performance evaluation of load control schemes in CDMA networks. The distinction between the following four classes of traffic models allows a first classification.

- Static models are models with a given number of active users with fixed position;

- In semi-static models, "snapshots" of active users are seen as realizations of spatial Poisson processes; these snapshots are used as the non-constrained traffic process on which one can evaluate (in)feasibility probabilities.

- in semi-dynamic models, users (or calls) arrive at a random location and last for some random duration; each user is motionless during its call; this is the "minimal" dynamic model where an admission control can be specified, and where blocking probabilities can be considered.

- In dynamic models, we have the same as above but customers may move during their calls; an admission and motion (or handoff) control can then be specified. Blocking and motion-cut probabilities can be evaluated.

The QoS indicators introduced for semi-static models in $[5,20,12,4]$ correspond to the probability that the SINR is less than some threshold, when users, modeled as a spatial Poisson point process, are all accepted. In [12] and [4] this indicator is called the outage probability. The authors of [20] call it the blocking probability, but as mentioned in [12], the term outage probability is more appropriate. We propose to make the following distinction between outage and infeasibility probabilities, both being defined for a semi-static model: the former is related to the event that the transmission quality of service is not attained for given transmission powers, whereas the latter corresponds to the situation when there is no solution to the power control problem.

The authors of [12] define the blocking probability in a semi-dynamic model and give simulation results, which show that the outage and blocking probabilities are different in general. In [3] it is argued that "the outage probability may easily be computed whereas the blocking probability, even in the particular case where a product-form is obtained, requires methods such as Monte-Carlo acceptancerejection technique or approximation techniques such as Erlang fixed point."

In analytical studies of the blocking probabilities in CDMA networks, the geometry of interferences specific to CDMA is often absent or seriously reduced. These studies make the distinction between blocking of new calls and of handoff calls. Examples of such studies are [15, 6], which consider a single cell and $[13,18]$, which consider a multi-cell scenario. In $[15,6]$, blocking probabilities are calculated via the classical Erlang formula. In [13], Erlang fixed point approximations are used to calculate blocking probabilities. In [18] explicit expressions of blocking probabilities are given for two limiting regimes of the dynamic model: no mobility and infinite mobility.

As already mentioned, the references $[2,1]$, take into account the geometry of interference and they evaluate the feasibility probabilities in a semi-static model within this setting.

\section{Decentralized up and downlink admission control in large CDMA networks}

In this section we recall briefly the decentralized admission control schemes for CDMA networks developed in $[2,1]$.

\section{$3.1 \quad$ Notation}

We will use the following notation: 


\subsubsection{Antenna locations and path loss}

- $\left\{Y^{u}\right\}_{u}$ denotes the locations of BS's; $Y^{u}$ denotes the location of the BS with index $u$;

- $S_{u}$ is set of mobiles served by BS $u$;

- $\left\{X_{m}^{u}\right\}_{m}$, with $m \in S_{u}$, denotes the locations of the mobiles served by BS $u$;

- $L(y, x)$ is the path-loss of signal on the path $y \rightarrow x$;

- $l_{\downarrow_{m}}^{u}=L\left(Y^{u}, X_{m}^{u}\right)$ is the path-loss on the downlink $Y^{u} \rightarrow X_{m}^{u}$;

- $l_{\uparrow}^{u}=L\left(X_{m}^{u}, Y^{u}\right)$ is the path-loss on the uplink $X_{m}^{u} \rightarrow Y^{u}$.

\subsubsection{Engineering parameters}

- $\alpha_{u}$ denotes the downlink (DL) orthogonality factor in BS $u$; let

$$
\alpha_{u v}= \begin{cases}\alpha_{u} & \text { if } u=v \\ 1 & \text { if } u \neq v\end{cases}
$$

- $\xi_{m}^{u}$ is the SINR threshold for user $X_{m}^{u} ; \quad \xi_{\downarrow_{m}}^{u}, \xi_{\uparrow_{m}^{u}}^{u}$ if it is necessary to distinguish between the downlink (DL) and the uplink (UL). Moreover, for each SINR $\xi$, we define a modified SINR $\xi^{\prime}$ by

$$
\xi_{\downarrow}^{u}=\frac{\xi_{\downarrow_{m}}^{u}}{1+\alpha_{u} \xi_{\downarrow_{m}}^{u}}, \quad \xi_{\uparrow_{m}^{\prime u}}^{u}=\frac{\xi_{\uparrow_{m}^{u}}^{u}}{1+\xi_{\uparrow_{m}^{u}}^{u}} .
$$

- $P_{\downarrow}^{u}$ is the power of the dedicated channel $u \rightarrow m$;

- $P_{\uparrow}^{u}$ is the power transmitted by mobile $m \rightarrow u$;

- $\tilde{P}^{u}$ denotes the maximal total power of BS $u$ (in the blocking rate calculation, we use also $\bar{P}_{\downarrow}$ to denote the average maximal power, which we assume not to depend on the BS index),

- $P^{\prime u}$ is the total power of the common channels $(\mathrm{CCH})$,

- $P^{u}=P^{\prime u}+\sum_{m} P_{\downarrow_{m}}^{u}$ is the total power transmitted by BS $u$;

- $\tilde{P}_{m}^{u}$ is the maximal power of mobile $m \in S_{u}$ (in the blocking rate calculation we use also $\bar{P}_{\uparrow}$ to denote the average maximal power that does not depend on the mobile index);

- $N$ is the external noise; $N^{u}, N_{m}^{u}$ are used for the noise at BS $u$ and at mobile $m \in S_{u}$, respectively (in the blocking rate calculation, we use also $\bar{N}_{\uparrow}, \bar{N}_{\downarrow}$ to denote the average external noise at the BS and mobile, respectively; we assume that these quantities do not depend on the index).

\subsection{Power Control with Power Constraints}

We now recall the power control problems with power constraints, so called feasibility problems. (We use equivalently power control and power allocation.) 


\subsubsection{Downlink}

We will say that the (downlink) power allocation with power limitations is feasible if there exist nonnegative and finite powers $P_{\downarrow}^{u}$, for all base stations $u$ and all mobiles $m$, which satisfy the following two conditions:

DL1. signal to interference and noise ratio at each mobile is larger than the threshold $\xi_{\downarrow}^{u}$, i.e.

$$
\frac{P_{\downarrow_{m}}^{u} / l_{\downarrow_{m}}^{u}}{N_{m}^{u}+\sum_{v} \alpha_{u v}\left(P^{\prime} v+\sum_{n \in S_{v}} P_{\downarrow_{n}}^{v}\right) / l_{l_{m}}^{v}} \geq \xi_{\downarrow_{m}}^{u},
$$

for all $u$ and $m \in S_{u}$;

DL2. the total power transmitted by each base station is not larger than its given limit $\sum_{m \in S_{u}} P_{\downarrow_{m}}^{u}+$ $P^{\prime} u \leq \tilde{P}^{u}$, for all $u$.

We will say that the (downlink) power allocation (without power limitations) is feasible if there exist nonnegative powers $P_{\downarrow_{m}}^{u}$ such that condition DL1 is satisfied.

\subsubsection{Uplink}

We will say that the (uplink) power control with power constraints is feasible if there exist finite nonnegative powers $P_{\uparrow}^{u}$ such that the following two conditions are satisfied:

UL1. signal to interference and noise ratio at each BS is larger than the threshold $\xi_{\uparrow_{m}}^{u}$, i.e.

$$
\frac{P_{\uparrow m}^{u} / l_{m}^{u}}{N^{u}+\sum_{v} \sum_{n \in S_{v}} P_{\uparrow_{n}^{v}}^{v} / l_{\uparrow}^{u}} \geq \xi_{\uparrow_{m}^{\prime}}^{u}
$$

for all $u, m \in S_{u}$.

UL2. the power transmitted by each mobile is not larger than its given limit $P_{\uparrow}^{u} \leq \tilde{P}_{m}^{u}$, for all $u$ and $m \in S_{u}$.

We will say that the (uplink) power control (without power constraints) is feasible if there exist nonnegative powers $P_{m}^{u}$ such that condition UL1 is satisfied.

\subsection{Decentralized Admission Control}

Assume the bit rates of all users (or equivalently all ${\downarrow_{m}}_{\downarrow_{m}}^{u}, \xi_{\uparrow_{m}}^{\prime u}$ parameters) to be specified. The admission control problem can then be posed as follows: for a given mobile population $\left\{m \in S_{u}\right\}$, for all BS $u$, check whether the (downlink) power allocation and the (uplink) power control with power constraints (defined in Section 3.2) are feasible. We now recall the decentralized sufficient conditions for feasibility developed in $[2,1]$.

\subsubsection{Downlink}

For user $m \in S_{u}$ define its downlink-power-control load with respect to BS $u$ by

$$
f_{\downarrow m}^{u}=\left(N_{m}^{u}+\sum_{v} \frac{\alpha_{u v} \tilde{P}^{v}}{l_{\downarrow_{m}}^{v}}\right) \frac{l_{\downarrow_{m}^{u}}^{u} \xi_{\downarrow_{m}}^{u}}{\tilde{P}^{u}} .
$$

Consider the two conditions

$$
\sum_{m \in S_{u}}\left(\sum_{v} \frac{\alpha_{u v} l_{\downarrow m}^{u} \xi_{\downarrow_{m}}^{u}}{l_{\downarrow_{m}}^{v}}\right)<1
$$


and

$$
\sum_{m \in S_{u}} f_{\downarrow m}^{u} \leq 1-\frac{P^{\prime} u}{\tilde{P}^{u}} .
$$

Note that (3.3) is equivalent to (3.4) with $\tilde{P}^{v}=\tilde{P}^{u}=\infty$. Consider the following algorithms.

\subsection{2 (Extended) Downlink Admission Control Protocol ((E-)DACP)}

Each BS checks periodically whether condition (3.3) (or (3.4)) is satisfied and, if not, enforces it by reducing the population $\left\{m: m \in S_{u}\right\}$ of its mobiles to some subset s.t. the inequality holds with the reduced population. When a new mobile user applies to some BS, the BS accepts it if the corresponding condition is satisfied with this additional user and rejects it otherwise.

It is shown in [1] that the application of DACP by all the BS's guarantees the global feasibility of the downlink power control problem DL1 without power constraints, whereas E-DACP guarantees in addition that the solution of the power allocation problem satisfies the maximal-power constraints DL2. Moreover, it was shown in [2] that under DACP, $P_{\downarrow_{m}}^{u}=\tilde{P}^{u} f_{\downarrow_{m}}^{u}, m \in S_{u}$, provides a finite solution to problem DL1.

\subsubsection{Uplink}

For user $m \in S_{u}$ define its uplink-power-control load with respect to BS $v$ as

$$
f_{\uparrow m}^{u v}=\frac{\xi_{\uparrow_{m}^{\prime u}}^{u} l_{m}^{u}}{l_{\uparrow m}^{v}}
$$

and its aggregated uplink-power-control load as

$$
f_{\uparrow}^{u}=\sum_{v} f_{\uparrow}^{u v}
$$

Consider the following conditions

$$
\sum_{m \in S_{u}} f_{\uparrow}^{u}<1
$$

and

$$
\sum_{m \in S_{u}} f_{\uparrow}^{u} \leq \theta_{u}
$$

where

$$
\theta_{u}=1-N^{u} \sup _{\substack{\text { all possible } \\ m \in S u}} \frac{\xi_{\uparrow_{m}^{\prime}}^{\prime^{u}} l_{\uparrow}^{u}}{\tilde{P}_{m}^{u}} ;
$$

The sup in (3.8) is taken over all possible locations for user $m \in S_{u}$ served by BS $Y^{u}$. Consider the following algorithms.

\subsection{4 (Simplified Extended) Uplink Admission Control Protocol ((SE-)UACP)}

Each BS checks periodically whether condition (3.7) (or (3.8)) is satisfied and, if not, enforces it by reducing the population $\left\{m: m \in S_{u}\right\}$ of its mobiles to some subset s.t. the inequality holds with the reduced population. When a new user applies to some $B S$, the $B S$ accepts it if the condition is satisfied with this additional user and rejects it otherwise.

It is shown in [1] that the application of the UACP by all BS's guarantees the global feasibility of the uplink power control problem UL1 without power constraints, whereas the application of SEUACP by all the BS's ensures the global feasibility of the uplink power control problem UL1 with power constraints UL2.

$\operatorname{RR} \mathrm{n}^{\circ} 5517$ 
These schemes are said to be decentralized in that each base station decides on admission based on the location of the mobiles in its cell and the location of other base stations but not on the location of mobiles outside its own cell.

\section{Blocking rates in decentralized admission control}

The decentralized admission control schemes of Section 3.3 prevent certain configurations of users in each cell from occurring. In order to quantify this phenomenon one can adopt two approaches, defining two QoS metrics for the schemes.

- A base-station-centric one, called in [1] the Cell Probability of Rejection (CPR), and also called here infeasibility probability. This consists in analyzing how often in an infinite network of base stations, an unconstrained (say Poisson) configuration of users cannot be entirely accepted by a cell due to the admission scheme under consideration.

- A user-centric one, called blocking rate associated with a given location in the cell, and which can be defined as the fraction of users arriving (say according to a birth-and-death process) at this location that cannot be accepted.

The two notions of QoS are closely related. This relation, as shown in the Appendix 6, is not bound to our particular admission control schemes, but is of more general nature and in a simplified scenario can be identified with the well-known Erlang loss formula. Namely, the complement of the CPR is the normalizing constant in the blocking rate formula.

In this section we will summarize and apply the results of the Appendix, in particular Proposition A.6 and Corollary A.7 to the CDMA networks operated under the decentralized admission control schemes of Section 3.3.

We first consider configurations of users in a typical cell of a given pattern of base stations. Assuming a simple (unconstrained) birth-and-death process of arrivals and departures in this cell, we estimate the feasibility probability. This can be further translated into the blocking probability of a typical user, via Proposition A.6 and Corollary A.7.

\subsection{Network Architecture and User Traffic}

In order to calculate feasibility and blocking probabilities we have to fix a network architecture and its parameters, as well as probabilistic assumptions concerning users.

\subsubsection{Infinite hexagonal network of BS's}

Denote by $\lambda_{B S}$ the mean number of BS's per unit of space. Each BS serves users in its cell defined as the set of locations in the plane which are closer to that BS than to any other BS. It is convenient to relate $\lambda_{B S}$ to the radius $R$ of the (virtual) disc whose area is equal to that of the area of the cell, by the formula

$$
\lambda_{B S}=1 /\left(\pi R^{2}\right) .
$$

With this definition in mind, we will sometimes call $R$ the radius of the cell. In the hexagonal model, the radius $R$ is related to the distance $\Delta$ between two adjacent BS's by $\Delta^{2}=2 \pi R^{2} / \sqrt{3}$. The BS's are located on the grid denoted on the complex plane by $\left\{Y^{u}: Y^{u}=\Delta\left(u_{1}+u_{2} e^{i \pi / 3}\right), u=\left(u_{1}, u_{2}\right) \in\right.$ $\left.\{0, \pm 1, \ldots\}^{2}\right\}$. The cell-pattern in this model is sometimes called honeycomb. Note that we consider the honeycomb on the whole plane. 


\subsubsection{Path-loss}

We model path-loss on distance $r$ by

$$
L(r)=(K r)^{\eta}
$$

where $\eta>2$ is the so-called path-loss exponent and $K>0$ is a multiplicative constant.

\subsubsection{Free SBD process of calls}

Fix one cell of the honeycomb described above, say that corresponding to BS 0 , located in $Y^{0}$; for simplicity we will omit the superscript 0 in what follows. Following the notation introduced in the Appendix, we denote this cell, considered as a subset of $\mathbb{R}^{2}$, by $\mathbb{D}$. We will model the process of call arrivals to and departures from $\mathbb{D}$ as a spatial birth-and-death (SBD) process: for a given subset $A \subset \mathbb{D}$, interarrival times to $A$ are independent exponential random variables with mean $1 / \lambda(A)$, where $\lambda(\cdot)$ is some given intensity measure of arrivals to $\mathbb{D}$ per unit of time. This allows the modeling of spatial hot spots. In homogeneous traffic conditions, we can take $\lambda(\mathrm{d} x)=\lambda \mathrm{d} x$, where $\lambda$ is the mean number of arrivals per unit of area and per unit of time. We assume that call holding times are independent exponential random variables with mean $\tau$. This description corresponds to the SBD process (see example A.4) with intensity of arrivals $\lambda(\cdot), r\left(\nu, \mathcal{A}_{x} \nu\right)=1$ and $r\left(\nu, \mathcal{D}_{x} \nu\right)=1 / \tau$ for $x \in \mathbb{D}$. Here $\nu$ is a point measure which characterizes the configuration of user locations $\left\{X_{m}\right\}$ in cell $\mathbb{D}$. In what follows, other characteristics of users and BS's such as powers $\tilde{P}^{0} \equiv \bar{P}_{\downarrow}, \tilde{P}_{m}^{0} \equiv \bar{P}_{\downarrow}$, SINR's $\xi_{\downarrow_{m}}^{0} \equiv \xi_{\downarrow}^{\prime}, \xi_{\uparrow_{m}}^{\prime 0} \equiv \xi_{\uparrow}^{\prime}$, noises $N_{m}^{0} \equiv \bar{N}_{\downarrow}, N^{0} \equiv \bar{N}_{\uparrow}$ and orthogonality factors $\alpha_{m v} \equiv \alpha$, are assumed to be constant for the sake of simplicity. Note that the SBD process described here models call arrivals and departures that are subject to no admission control. Thus we call it the free process of calls.

Denote by $\mathbb{M}$ the set of all possible configurations $\nu=\left\{X_{m}\right\}$ of users in cell $\mathbb{D}$, i.e. $\mathbb{M}=\left\{\nu: X_{m} \in\right.$ $\mathbb{D}, m=1, \ldots, n$, for some $n \geq 0\}$ in this free process.

Note that the admission control protocols DACP, E-DACP, UACP, SE-UACP described in Section 3.3, applied by the BS $Y^{0}$, only allow user configurations $\nu=\left\{X_{m}\right\} \in \mathbb{M}$ that satisfy condition

$$
\sum_{m} \tilde{f}\left(X_{m}\right)<C
$$

for some non-negative function $\tilde{f}$ defined on $\mathbb{D}$ and some constant $C$. Moreover, for each protocol described in Section 3.3, the function $\tilde{f}$ is some linear operator of the following function

$$
f(x)=\sum_{v \neq 0} \frac{L(|x|)}{L\left(\left|Y^{v}-x\right|\right)}
$$

that depends only on the geometry of the BS network and the path-loss model. Adopting point-process notation (see Appendix A.1.1) we can rewrite condition (4.2) in the following form

$$
\int_{\mathbb{D}} \tilde{f}(x) \nu(\mathrm{d} x)<C
$$

We will denote the set of possible configurations $\nu \in \mathbb{M}$ that satisfy condition (4.3) by $\tilde{\mathbb{M}}$ and call it the set of feasible configurations for the admission control protocol in question.

\subsubsection{Loss process of calls}

Consider now the situation where the process of arrivals is as above but subject to the admission control scheme: from a given configuration, a new call is accepted if the new configuration still satisfies (4.3) and rejected otherwise, and where the holding times of accepted calls are exponential as above. Define the blocking rate $b_{x}$ at a given point $x \in \mathbb{D}$ as the fraction of users that are rejected in the stationary regime of this loss system in some infinitesimal neighborhood of location $x$ (see Appendix A.2 for the precise definition).

$\operatorname{RR} \mathrm{n}^{\circ} 5517$ 


\subsection{Feasibility Probability and Blocking Rates}

Denote by $\Pi$ the distribution of the Poisson point process on $\mathbb{D}$ with mean measure $\tau \lambda(\cdot)$. We have the following main result concerning feasibility probability and blocking rates.

Theorem 4.1 (i) The stationary distribution of the free SBD process of calls described in Section 4.1 .3 is $\Pi$ and so the feasibility probability (i.e. the probability that the a given realization of the stationary free process of calls in $\mathbb{D}$ satisfies condition (4.3) is given by $\Pi(\tilde{\mathbb{M}})$.

(ii) The blocking rates of the loss process described in Section 4.1 .4 are equal to

$$
b_{x}=\frac{\Pi\left\{\nu: C-\tilde{f}(x) \leq \int_{\mathbb{D}} \tilde{f}(z) \nu(d z)<C\right\}}{\Pi(\tilde{\mathbb{M}})} .
$$

The proof of the above result is given in the Appendix (see Corollary A.7). It is a special case of a more general result (see Proposition A.6) concerning a general SBD process, where the free process of calls can be dependent in some probabilistic way on the current state of the system. In this more general case, the Poisson distribution $\Pi$ should be replaced by some Gibbs distribution based on $\Pi$. Moreover, one can consider a more general form of feasibility condition.

Note that the formula (4.4) has the form of the Erlang loss formula (1.1). In particular, the feasibility probability $\Pi(\tilde{\mathbb{M}})$ is the normalizing constant. The complement of the feasibility probability was called the cell probability of rejection in [1].

\subsection{Approximations of Blocking Rates}

In order to calculate the blocking rates via formula (4.4) one needs to know the distribution function of

$$
I=\int_{\mathbb{D}} \tilde{f}(z) \nu(\mathrm{d} z)
$$

under П. For this, we use the Gaussian approximations developed in [1]. More precisely, we approximate the sum $I$ by the Gaussian random variables with mean and variance equal to those of $I$ under П. The quality of this approximation, theoretically justified by the Central Limit Theorem, has already been validated in [1] by comparison with simulations, in the case of the feasibility probability $\Pi(\tilde{\mathbb{M}})=\Pi(I \leq C)$. Denote by $\epsilon$ and $\sigma^{2}$, respectively, the mean and the variance of $I$ under $\Pi$. Using the same approach we get the following blocking rate approximations

$$
b_{x} \approx \frac{Q((C-\tilde{f}(x)-\epsilon) / \sigma)-Q((C-\epsilon) / \sigma)}{1-Q((C-\epsilon) / \sigma)},
$$

where $Q(z)=1 / \sqrt{2 \pi} \int_{z}^{\infty} e^{-t^{2} / 2} \mathrm{~d} t$ is the Gaussian tail distribution function. Moreover, for small $\tilde{f}(x) / \sigma$, the following approximation can by justified

$$
b_{x} \approx \frac{\tilde{f}(x) e^{-(C-\epsilon)^{2} /\left(2 \sigma^{2}\right)}}{\sqrt{2 \pi} \sigma(1-Q((C-\epsilon) / \sigma))} .
$$

For completeness, we recall below the formulas developed in [1] for $\epsilon$ and $\sigma^{2}$, corresponding to the admission control protocols DACP, E-DACP, UACP, SE-UACP. They rely on the following approximation for $f(x)$ proposed in [8]

$$
\begin{aligned}
f(x) & \approx \zeta(\eta-1) L(|x|) \\
& \times\left(\frac{1}{L(\Delta-|x|)}+\frac{1}{L(\Delta+|x|)}+\frac{4}{L\left(\sqrt{\Delta^{2}+|x|^{2}}\right)}\right)
\end{aligned}
$$


for $|x| \leq R$, where $\zeta(s)=\sum_{n=1}^{\infty} 1 / n^{s}$ is the Riemann zeta function (recall that $\Delta$ is the distance between two adjacent BS's in the hexagonal network and $R$ is the radius of the disc with area equal to that of the cell).

In the following expressions, we use some constants, which were calculated numerically under the above assumptions: $\bar{f} \approx 0.9365 /(\eta-2), \bar{g} \approx 1 /(1+\eta / 2), \overline{f^{2}} \approx 0.2343 /(\eta-2)+1.2907 /(\eta-2)^{2}, \overline{l f} \approx$ $0.6362 /(\eta-2)$. We assume a homogeneous traffic scenario and in these expressions, $\bar{M}=\tau \lambda /\left(\pi R^{2}\right)$ is the mean number of users of the free process in the cell.

\section{DACP}

$$
\begin{aligned}
C & =1, \\
\epsilon & =\bar{M} \xi_{\downarrow}^{\prime}(\alpha+\bar{f}), \\
\sigma^{2} & =\bar{M} \xi_{\downarrow}^{\prime 2}\left(\alpha^{2}+2 \alpha \bar{f}+\overline{f^{2}}\right), \\
\tilde{f}(x) & =\xi_{\downarrow}^{\prime}(\alpha+f(x)) .
\end{aligned}
$$

\section{E-DACP}

$$
\begin{aligned}
C= & 1-\frac{P^{\prime}}{\bar{P}_{\downarrow}}, \\
\epsilon= & \bar{M} \xi_{\downarrow}^{\prime}\left(\alpha+\bar{f}+L(R) \bar{N}_{\downarrow} \bar{g} / \bar{P}_{\downarrow}\right), \\
\sigma^{2}= & \bar{M}{\xi_{\downarrow}^{\prime}}^{2}\left(\bar{N}_{\downarrow}^{2} L^{2}(R) \bar{g}(2 \eta) / \bar{P}_{\downarrow}^{2}+\alpha^{2}+\bar{f}^{2}\right. \\
& \left.+2\left(\alpha \bar{f}+\bar{N}_{\downarrow} L(R)(\alpha \bar{g}+\overline{l f}) / \bar{P} \downarrow\right)\right), \\
\tilde{f}(x)= & \xi_{\downarrow}^{\prime}\left(\bar{N}_{\downarrow} / \bar{P}_{\downarrow}+\alpha+f(x)\right) .
\end{aligned}
$$

UACP

$$
\begin{aligned}
C & =1, \\
\epsilon & =\bar{M} \xi_{\uparrow}^{\prime}(1+\bar{f}), \\
\sigma^{2} & =\bar{M} \xi_{\downarrow}^{\prime 2}\left(\overline{f^{2}}+1+2 \bar{f}\right), \\
\tilde{f}(x) & =\xi_{\uparrow}^{\prime}(1+f(x)) .
\end{aligned}
$$

SE-UACP

$$
\begin{aligned}
C & =1-\overline{N_{\uparrow}} L(R) \xi_{\uparrow}^{\prime} / \bar{P}_{\uparrow}, \\
\epsilon & =\bar{M} \bar{\xi}_{\uparrow}^{\prime}(1+\bar{f}), \\
\sigma^{2} & =\bar{M}{\xi_{\uparrow}^{\prime}}^{2}\left(\overline{f^{2}}+1+2 \bar{f}\right), \\
\tilde{f}(x) & =\xi_{\uparrow}^{\prime}(1+f(x)) .
\end{aligned}
$$

\section{$5 \quad$ Numerical results}

In this section we will give a few numerical examples.

\subsection{Model Specification}

We will study blocking rates for the different admissions protocols with different values of the cell radius $R$ and mean number of customers per cell $\bar{M}$. The following values are fixed for the study. 


\subsubsection{Path Loss}

$\eta=3.38, K=8667$.

\subsubsection{Physical layer parameters}

$\alpha=0.4, \xi_{\downarrow}=-16 \mathrm{~dB}, \xi_{\uparrow}=-18 \mathrm{~dB}, \bar{N}_{\uparrow}=-105 \mathrm{dBm}, \bar{N}_{\downarrow}=-103 \mathrm{dBm}$ (external noise at the BS and user, respectively), $\bar{P}_{\downarrow}=52 \mathrm{dBm}, \bar{P}_{\uparrow}=33 \mathrm{dBm}$ (maximal powers of BS and mobile, respectively, including antenna gains and losses), $P^{\prime}=42.73 \mathrm{dBm}$. The above values correspond to the UMTS specification [10].

In what follows we will study the infeasibility probability $P=\Pi(I \leq C)$, the blocking rates $b_{x}$ and the average blocking rate $\bar{b}$ defined as follows

$$
\bar{b}=\frac{1}{\pi R^{2}} \int_{|x| \leq R} b_{x} \mathrm{~d} x
$$

for the four admission control schemes DACP, UACP, E-DACP, SE-UACP. Since our blocking formulas are rotation invariant, we will slightly abuse the notation writing $b_{r}=b_{x}$ for $|x|=r$. Moreover, we will plot $b_{r}$ as the function of the normalized distance $r / \Delta$, where $\Delta$ is the distance between two adjacent BS's. Note that $R=\Delta \sqrt{\sqrt{3} /(2 \pi)}$ and thus the value $R / \Delta \approx 0.525$ corresponds to the normalized distance from the cell edge to the BS.

\subsection{No Power Limitation}

We first consider schemes which do not take power limitations into account. Then the infeasibility probability $P=P(I \leq C)$ and the blocking rates $b_{r}=b_{r}(\bar{M}), \bar{b}=\bar{b}(\bar{M})$ depend on the mean number $\bar{M}$ of customers per cell and do not depend on the cell radius $R$.

\subsubsection{DACP}

Figure 1 shows blocking rates $b_{r}(\bar{M})$ calculated by the two approximations (4.5) and (4.6), for fixed $\bar{M}=27$, as functions of the normalized distance $r / \Delta$. Note that both approximations give similar numerical values; however, their values differ more at the cell edge, where the difference attains about $30 \%$. The blocking rate $b_{r}$ increases with the distance $r$ of the user to its base station and at the cell edge $r / \Delta \approx 0.525$, it is about 10 times bigger than at the cell center. Figure 2 shows the average blocking rate $\bar{b}(\bar{M})$, the blocking rate at cell edge $b_{R}(\bar{M})$, both calculated via (4.5), and the infeasibility probability $P(\bar{M})$ as functions of the mean number $\bar{M}$ of users per cell.

\subsubsection{UACP}

Figure 3 shows the blocking rates $b_{r}(\bar{M})$ calculated by the two approximations (4.5) and (4.6), as functions of the normalized distance $r / \Delta$ for fixed $\bar{M}=27$. Again, both approximations give similar numerical values. The blocking rate $b_{r}$ increases with the distance $r$ of the user to its base station and at the cell edge $R / \Delta \approx 0.525$, it is about 4 times bigger that at the cell center. Figure 4 shows the average blocking rate $\bar{b}(\bar{M})$, the blocking rate at cell edge $b_{R}(\bar{M})$, both calculated via (4.5), and the infeasibility probability $P(\bar{M})$ as functions of the mean number $\bar{M}$ of users per cell.

\subsection{With Power Limitation}

We will now take into account the maximal power limitation. In this case, the infeasibility probability $P=P(\bar{M}, R)$ and blocking rates $b_{r}=b_{r}(\bar{M}, R), \bar{b}=\bar{b}(\bar{M}, R)$ depend on the cell radius $R$ too. 
$b_{r}$

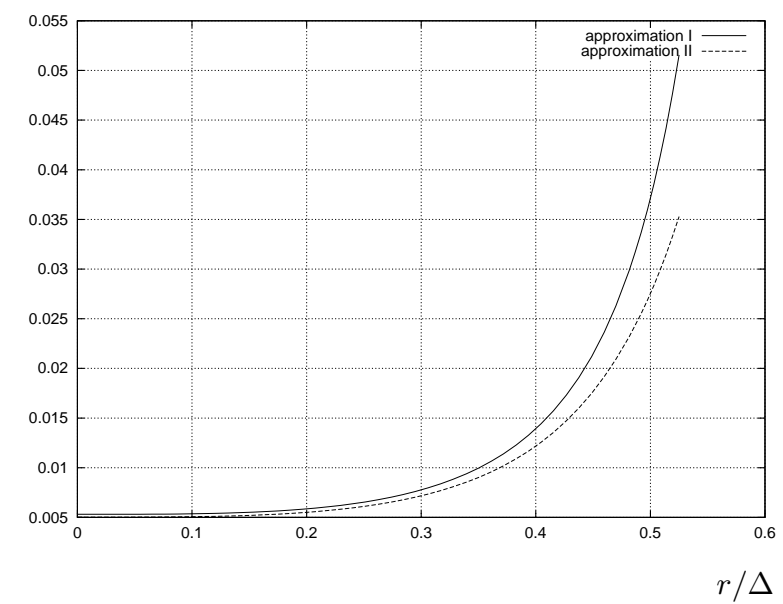

Figure 1: DACP; blocking rates $b_{r}(\bar{M})$ calculated via approximations (4.5) - I and (4.6) II as functions of the normalized distance $r / \Delta$ for $\bar{M}=27$.

$b_{r}$

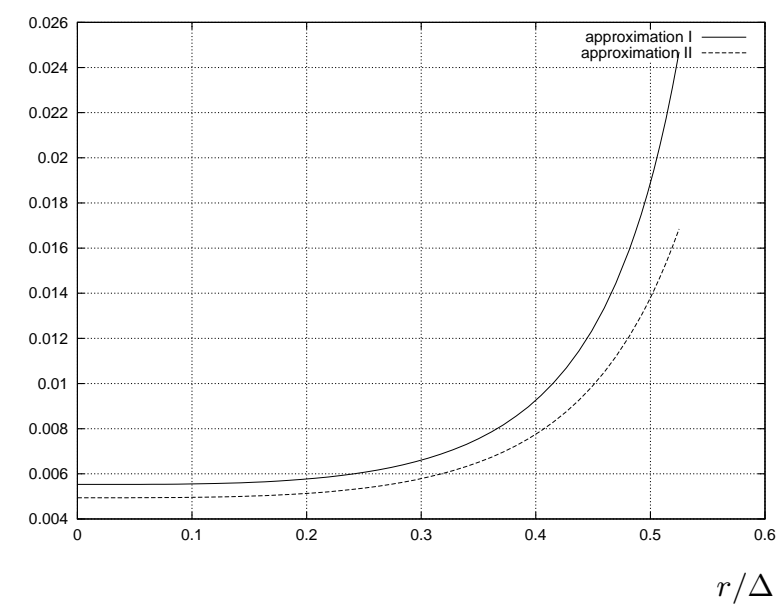

Figure 3: UACP; blocking rates $b_{r}(\bar{M})$ calculated via approximations (4.5) - I and (4.6) II as functions of the normalized distance $r / \Delta$ for $\bar{M}=27$. rate, probability

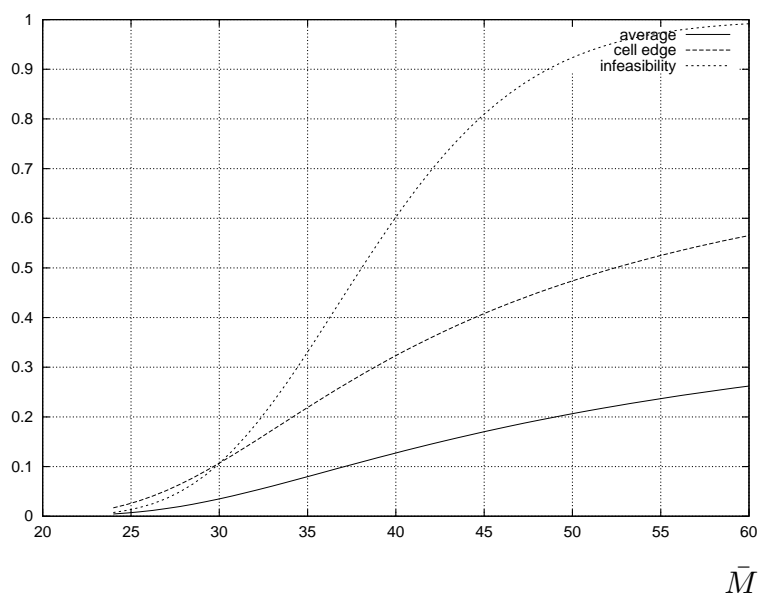

Figure 2: DACP; Average blocking rate, the blocking rate at cell edge and the infeasibility probability as functions of the mean number of users $\bar{M}$ per cell.

rate, probability

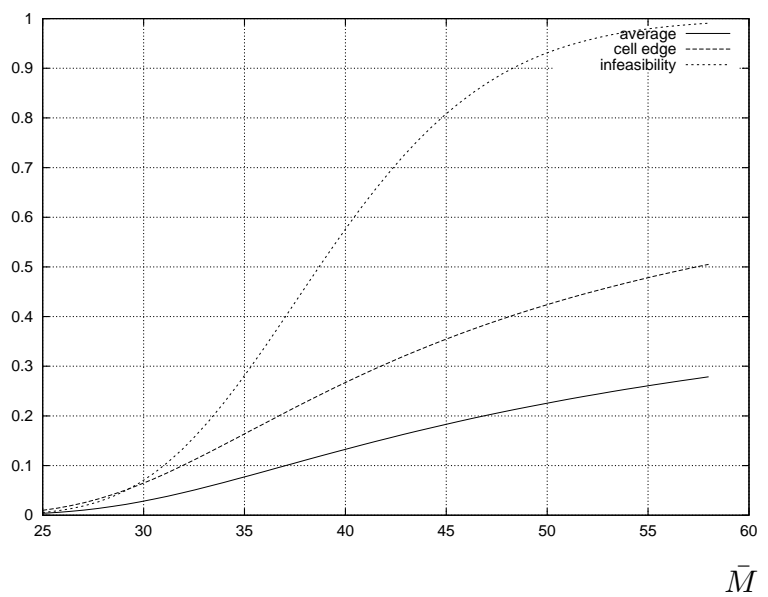

Figure 4: UACP; Average blocking rate, the blocking rate at cell edge and the infeasibility probability as functions of the mean number of users $\bar{M}$ per cell.

\subsubsection{E-DACP}

Figure 5 shows the average blocking rate $\bar{b}(\bar{M}, R)$, the blocking rate at cell edge $b_{R}(\bar{M}, x)$, both calculated via (4.5), and the infeasibility probability $P(\bar{M}, R)$ as functions of $\bar{M}$ for $R=1,3,5 \mathrm{~km}$. Note that $\bar{b}(\bar{M}, R)$ is about one third of $b_{R}(\bar{M}, R)$. 


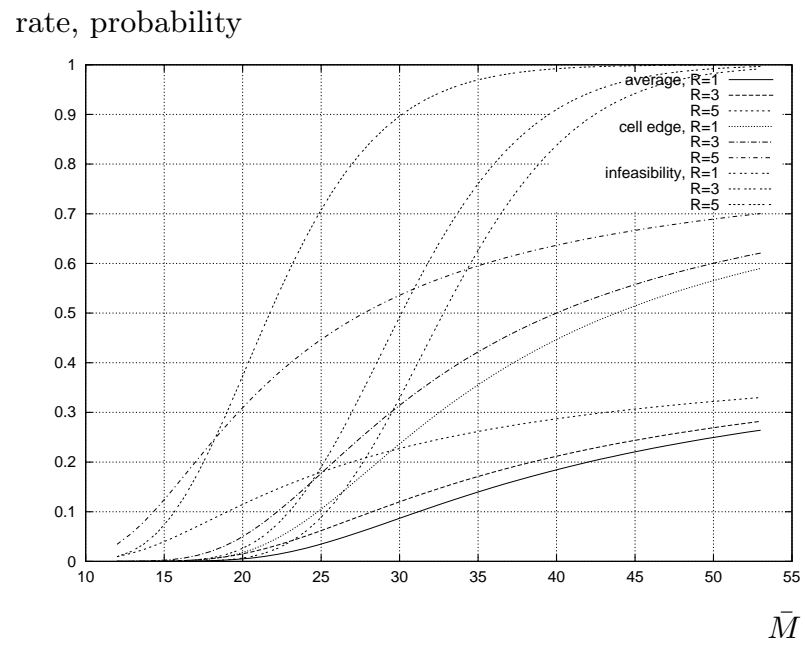

Figure 5: E-DACP; from bottom to top: average blocking rate, the blocking rate at cell edge and the infeasibility probability as functions of the mean number of users $\bar{M}$ per cell for $R=$ $1,3,5 \mathrm{~km}$. rate, probability

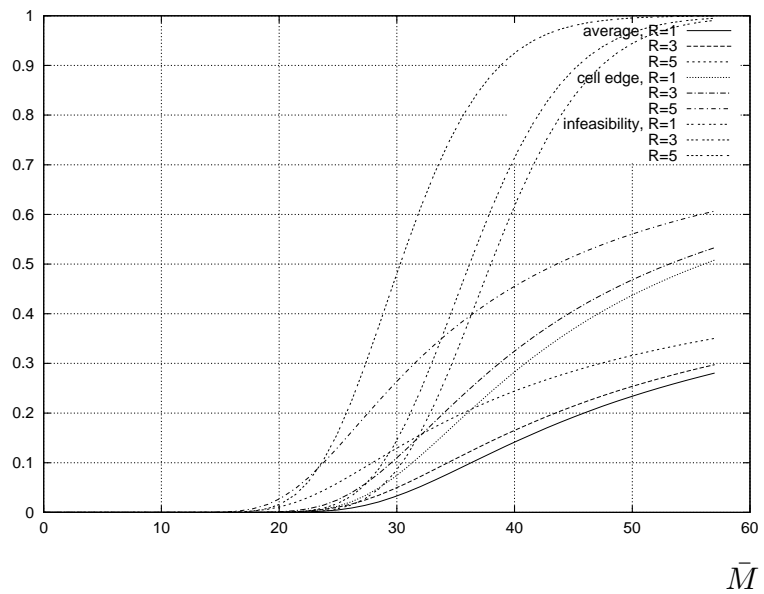

Figure 6: SE-UACP; from bottom to top: average blocking rate, the blocking rate at cell edge and the infeasibility probability as functions of the mean number of users $\bar{M}$ per cell for $R=$ $1,3,5 \mathrm{~km}$.

\subsubsection{SE-UACP}

Figure 6 shows the average blocking rate $\bar{b}(\bar{M}, R)$, the blocking rate at cell edge $b_{R}(\bar{M}, x)$ both calculated via (4.5), and the infeasibility probability $P(\bar{M}, R)$ as functions of $\bar{M}$ for $R=1,3,5 \mathrm{~km}$. Results are similar to those for the downlink.

Note that in all the above cases, the infeasibility probability is in general different from blocking rates.

\section{Concluding Remarks}

We have given an analytic expression for the blocking probability in CDMA networks in function of the cell radius, the antenna type, channel throughput (or equivalently SINR threshold), and offered traffic. We have also shown that this notion is closely related to the infeasibility probability introduced in earlier papers. The infeasibility probability is easy to calculate theoretically, but it cannot be easily measured in an existing network, whereas the blocking probability may easily be measured in the field. The relationship between these two notions thus makes it possible to start some statistical validations of the closed form expressions that have been obtained for both of them. Orange already implemented our analytical expression for the blocking probability in its dimensioning tools.

For simplicity we have assumed that the SINR required by all users were all the same. However our approach easily extends to multiclass SBD processes. It could also be extended to hexagonal networks with directional antennas and sectoring. Joint feasibility (and blocking) on the uplink and the downlink can also be treated in the same manner. Other useful extensions would concern macrodiversity and random fading.

In this paper, we considered the semi-dynamic model with no user mobility. In principle we could use the idea on the truncation of a general spatial queueing process introduced in [7], to study the model with user mobility. However, the truncated Markov model is not very realistic in this context as users that are denied service in a new location leave the system rather than stay blocked in the previous location. We will analyze motion-cut probabilities in a separate study. 


\section{Acknowledgment}

The second author is partially supported by KBN grant 2 P03A 02023.

\section{Appendix: Mathematical background}

In this section we develop mathematical tools for the spatio-temporal analysis of large networks with blocking. The context is more general than the CDMA setting described in this paper. In particular we will introduce notation and general notions of infeasibility and blocking rates.

\section{A.1 Preliminaries}

\section{A.1.1 Point process}

Very much as in [16], we will consider a system in which units take place in a complete, separable metric space $\mathbb{D}$, where they are processed. Typically $\mathbb{D}$ would be a bounded subset of the plane $\mathbb{R}^{2}$. If $\mathbb{D}$ is a finite set of points, the system is a discrete network. In the general case, we will represent the state of the system by a finite counting measure $\nu$ on $\mathbb{D}$. Suppose that $x_{1}, \ldots, x_{k} \in \mathbb{D}$ are the locations of units (or points). These units can be described by a counting measure $\nu$ on $\mathbb{R}^{2}$ defined by

$$
\nu(A)=\sum_{i=1}^{k} \varepsilon_{x_{i}}(A)
$$

for $A \in \mathcal{E}$, where $\varepsilon_{x}$ is a Dirac measure with unit mass at $x$, i.e. $\varepsilon_{x}(A)=1$ if $x \in A$ and 0 otherwise. As a simple consequence of this notation we have for a given real valued function $f$ on $\mathbb{R}^{2}: \int f(x) \nu(\mathrm{d} x)=$ $\sum_{i=1}^{k} f\left(x_{i}\right)$.

A random configuration $N$ of units at a given time, will be modeled by a point process that is a measurable mapping from some given probability space to the state space $\mathbb{M}$ of all finite counting measures on $\mathbb{D}$ (with the smallest $\sigma$-algebra $\mathcal{M}$ making the mappings $\mathbb{M} \ni \nu \mapsto \nu(B)$ for all $B \in \mathcal{E}$ measurable).

The mean measure $\lambda(\cdot)$ of the point process $N$ is defined as $\lambda(B)=\mathbf{E}[N(B)], B \in \mathcal{E}$; it represents the expected numbers of units present in subsets of $\mathbb{D}$.

Here are two examples of point processes.

Example A.1 The most prominent point processes are Poisson processes defined as follows: $N$ is Poisson with mean measure $\lambda$ if for each $A \subset \mathbb{D}$ the random variable $N(A)$ is Poisson with mean $\lambda(A)$ and for all mutually disjoint $A_{i} \subset \mathbb{D}$ the random variables $N\left(A_{i}\right), \ldots, N\left(A_{k}\right)$ are independent.

Example A.2 Another important class of distributions of point process is that of Gibbs processes. For a given nonnegative function $\overline{\mathcal{E}}: \mathbb{M} \rightarrow \mathbb{R}_{+}\left(\mathbb{R}_{+}\right.$denotes the set of non-negative real numbers) and a mean measure $\lambda$ on $\mathbb{D}$, the Gibbs distribution on $\mathbb{M}$, with energy function $\overline{\mathcal{E}}$ and Poisson weight process $N$ of mean measure $\lambda$, is the distribution $\Pi$ on $\mathbb{M}$ defined by

$$
\Pi(\Gamma)=Z^{-1} \mathbf{E}[1(N \in \Gamma) \overline{\mathcal{E}}(N)],
$$

where $Z=E[\overline{\mathcal{E}}(N)]$ is the normalizing constant called also partition function or statistical sum. The energy function can often be expressed as follows

$$
-\log (\overline{\mathcal{E}}(\nu))=\sum_{k=1}^{\nu(\mathbb{D})} \mathcal{E}\left(x_{k}, \sum_{i=1}^{k-1} \varepsilon_{x_{i}}\right),
$$

where $\nu=\sum_{i=1}^{\nu(\mathbb{D})} \varepsilon_{x_{i}}$, and where $\mathcal{E}$ is called the local energy function. 


\section{A.1.2 Measure-valued stochastic processes}

We will model the temporal evolution of the system of units by a Markov jump process $\left\{N_{t} ; t \geq 0\right\}$ taking values in the state space $\mathbb{M}$ of all finite counting measures on $\mathbb{D}$. The process $\left\{N_{t} ; t \geq 0\right\}$ is completely defined by its generator

$$
q(\nu, \Gamma)=\lim _{t \searrow 0} t^{-1} \mathbf{P}\left(N_{t} \in \Gamma \backslash\{\nu\} \mid N_{0}=\nu\right), \quad \nu \in \mathbb{M}, \Gamma \in \mathcal{M}
$$

We will always assume that $\left\{N_{t} ; t \geq 0\right\}$ is ergodic and will denote by $\Pi$ its limiting distribution satisfying

$$
\lim _{t \rightarrow \infty} \sup _{\Gamma \in \mathcal{M}}\left|\mathbf{P}\left(N_{t} \in \Gamma\right)-\Pi(\Gamma)\right|=0 .
$$

It is also an invariant measure in the sense that it satisfies the following global balance equation

$$
q(\nu, \mathbb{M}) \Pi(\mathrm{d} \nu)=\int_{\mathbb{M}} q(\mu, \mathrm{d} \nu) \Pi(\mathrm{d} \mu) .
$$

Fix a subset $\tilde{\mathbb{M}} \subset \mathbb{M}$ of the state space. Let $\{\tilde{N} ; t \geq 0\}$ be a Markov process on $\tilde{\mathbb{M}}$ with transition kernel $\tilde{q}(\nu, \Gamma)=q(\nu, \Gamma \cap \tilde{\mathbb{M}})$. The restricted process $\left\{\tilde{N}_{t} ; t \geq 0\right\}$ associated to $\tilde{q}$ might be seen as evolving in the same way as $\left\{N_{t} ; t \geq 0\right\}$ with the exception that all the transitions from a state $\nu \in \tilde{\mathbb{M}}$ to a state $\mu \in \mathbb{M} \backslash \tilde{\mathbb{M}}$ are "blocked", which means that the process remains in the state $\nu$ and continues its evolution driven by $q$. In what follows, we will assume that the restricted process is also ergodic and has for limiting distribution $\tilde{\Pi}$ the truncation of $\Pi$ to $\tilde{\mathbb{M}}$. This truncation property does not always hold, and one simple sufficient condition for it to hold is as follows (cf Proposition 3.14 in [16]):

Lemma A.3 The stationary distribution $\tilde{\Pi}$ of the restricted process $\left\{\tilde{N}_{t} ; t \geq 0\right\}$ is given by

$$
\tilde{\Pi}(\Gamma)=\frac{\Pi(\Gamma \cap \tilde{\mathbb{M}})}{\Pi(\tilde{\mathbb{M}})}
$$

if and only if $\Pi$ satisfies the following balance equation

$$
q(\nu, \tilde{\mathbb{M}}) \Pi(d \nu)=\int_{\tilde{\mathbb{M}}} q(\mu, d \nu) \Pi(d \mu), \quad \nu \in \tilde{\mathbb{M}} .
$$

This truncation property holds in particular if $\left\{N_{t} ; t \geq 0\right\}$ is reversible on $\tilde{\mathbb{M}}$ or $\mathbb{M} \backslash \tilde{\mathbb{D}}$ with respect to $\Pi$, meaning that $q$ satisfies the following detailed balance equation

$$
q(\nu, d \mu) \Pi(d \nu)=q(\mu, d \nu) \Pi(d \mu)
$$

for, either $\nu, \mu \in \tilde{\mathbb{M}}$ or $\nu, \mu \in \mathbb{M} \backslash \tilde{\mathbb{M}}$.

Here is an example of measure valued stochastic process that we will work with.

Example A.4 A spatial birth-and-death process (SBD) is a Markov measure-valued process on $\mathbb{M}$, which changes at isolated instants of time by either the birth of a new point $x$ that is added to the pattern $\nu$, or by the death of a point $y$ that is deleted form $\nu$. More precisely, define the generator of the SBD as follows

$$
\begin{aligned}
q(\nu, \Gamma)= & \int_{\mathbb{D}} r\left(\nu, \mathcal{A}_{y} \nu\right) 1\left(\mathcal{A}_{y} \nu \in \Gamma\right) \lambda(\mathrm{d} y) \\
& +\int_{\mathbb{D}} r\left(\nu, \mathcal{D}_{x} \nu\right) 1\left(\mathcal{D}_{x} \nu \in \Gamma\right) \nu(\mathrm{d} x),
\end{aligned}
$$

where 
- $\lambda(A)(A \subset \mathbb{D})$ is a finite measure on the state space $\mathbb{D}$ that is supposed to describe the intensity of the arrivals to the network in the absence of restriction.

- $\mathcal{A}_{y}, \mathcal{D}_{x}(x, y \in \mathbb{D})$ are, respectively, birth and death operators on the space $\mathbb{M}$ : for $\nu \in \mathbb{M}, x, y \in \mathbb{D}$

$$
\begin{aligned}
& \mathcal{A}_{y} \nu=\nu+\varepsilon_{y}, \\
& \mathcal{D}_{x} \nu=\nu-\varepsilon_{x} \quad \text { defined only if } \nu(\{x\}) \geq 1 .
\end{aligned}
$$

- $r(\nu, \mu)<\infty(\nu \in \mathbb{M})$ is a birth-death rate; it is supposed to describe the rate at which a single unit is "attracted" by configuration $\nu$ to take location $y$ or "repulsed" form its location $x$ in this configuration, with the repulsion and the attraction possibly being dependent on the entire configuration $\nu$. We implicitly assume that $r(\nu, \mu)=0$ unless $\mu=\mathcal{A}_{y} \nu$ or $\mu=\mathcal{D}_{x} \nu$.

We call $r\left(\nu, \mathcal{A}_{x} \nu\right) \lambda(\mathrm{d} x)$ the intensity of births and $r\left(\nu, \mathcal{D}_{x} \nu\right)$ the intensity of deaths. Under suitable conditions the SBD process is non-explosive, ergodic and converges to the equilibrium distribution. For our purpose it suffices to assume that

$$
\sup _{\nu \in \mathbb{M}} \int_{\mathbb{D}} r\left(\nu, \mathcal{A}_{x} \nu\right) \lambda(\mathrm{d} x)<\infty
$$

in order to guarantee that the SBD process is non-explosive (see Th. 5.1 in [14]). If moreover

$$
\inf _{o \neq \nu \in \mathbb{M}, x \in \mathbb{D}} r\left(\nu, \mathcal{D}_{x} \nu\right)>0
$$

where $o$ denotes the null measure, then the SBD process is ergodic (see see Th. 7.1 in [14]). If in addition the following condition is satisfied

$$
\frac{r\left(\nu, \mathcal{A}_{x} \nu\right) r\left(\mathcal{A}_{x} \nu, \mathcal{A}_{y} \mathcal{A}_{x} \nu\right)}{r\left(\mathcal{A}_{x} \mathcal{A}_{y} \nu, \mathcal{A}_{y} \nu\right) r\left(\mathcal{A}_{y} \nu, \nu\right)}=\frac{r\left(\nu, \mathcal{A}_{y} \nu\right) r\left(\mathcal{A}_{y} \nu, \mathcal{A}_{x} \mathcal{A}_{y} \nu\right)}{r\left(\mathcal{A}_{y} \mathcal{A}_{x} \nu, \mathcal{A}_{x} \nu\right) r\left(\mathcal{A}_{x} \nu, \nu\right)}
$$

for all $x, y \in \mathbb{D}$ and $\nu \in \mathbb{M}$, then the SBD process is reversible and its equilibrium distribution $\Pi$ is the Gibbs distribution with local energy

$$
\mathcal{E}(x, \nu)=-\ln \left(\frac{r\left(\nu, \mathcal{A}_{x} \nu\right)}{r\left(\mathcal{A}_{x} \nu, \nu\right)}\right) .
$$

based on the Poisson weight process with mean measure $\lambda(\cdot)$. See $[19,17]$.

\section{A.2 Feasibility and Blocking Rates for SBD Processes, Spatial Erlang Formula}

Consider an ergodic SBD process $\left\{N_{t} ; t \geq 0\right\}$ on $\mathbb{M}$ with stationary distribution $\Pi$. Consider its restriction $\left\{\tilde{N}_{t} ; t \geq 0\right\}$ to the state space $\tilde{\mathbb{M}}$ and assume that is also stationary, ergodic and with invariant distribution the truncation of $\Pi: \tilde{\Pi}(\cdot)=\Pi(\cdot \cap \tilde{\mathbb{M}}) / \Pi(\tilde{\mathbb{M}})$. We call $\Pi(\tilde{\mathbb{M}})$ the feasibility probability.

For a given set $B \in \mathbb{D}$, denote by $\tau_{B}$ the following ergodic frequency of births of the process $\left\{N_{t} ; t \geq 0\right\}$ in $B$, i.e. the a.s. limit

$$
\tau_{B}=\lim _{t \rightarrow \infty} \frac{1}{t} \sum_{O \leq s \leq t} 1\left(N_{s}=\mathcal{A}_{y} N_{s-}, y \in B\right) .
$$

Similarly, denote by $\tilde{\tau}_{B}$ the ergodic frequency of births of the process $\left\{\tilde{N}_{t} ; t \geq 0\right\}$ in $B$. 
Lemma A.5 We have

$$
\begin{aligned}
\tau_{B} & =\int_{B} \mathbf{E}^{\Pi}\left[r\left(N, \mathcal{A}_{y} N\right)\right] \lambda(d y) \\
\tilde{\tau}_{B} & =\int_{B} \mathbf{E}^{\tilde{\Pi}}\left[r\left(N, \mathcal{A}_{y} N\right) 1\left(\mathcal{A}_{y} N \in \tilde{\mathbb{M}}\right)\right] \lambda(d y) .
\end{aligned}
$$

Proof: By ergodicity, since $\Pi$ is the stationary distribution of $\left\{N_{t} ; t \geq 0\right\}$

$$
\tau_{B}=\mathbf{E}^{\Pi}\left[\sum_{0 \leq s \leq 1} 1\left(N_{s}=\mathcal{A}_{y} N_{s-}, y \in B\right)\right] .
$$

By Lévy's formula (see e.g. Corollary 7.5.3, p. 232 in [11] whose proof can be extended to our measure valued process)

$$
\tau_{B}=\int_{B} \mathbf{E}^{\Pi}\left[r\left(N, \mathcal{A}_{y} N\right)\right] \lambda(\mathrm{d} y),
$$

which completes the proof for the first equality. The same line of thought shows the second equality.

We define the acceptance ratio $a_{B}$ associated with the set $B$ as the ratio $\tilde{\tau}_{B} / \tau_{B}$. By Lemma A.5, $\tau_{B}$ and $\tilde{\tau}_{B}$ can be seen as measures on $\mathbb{D}$ and $\tilde{\tau}$ is absolutely continuous with respect to $\tau$. We will define the acceptance rate $a_{y}$ for the births at $y$ by the Radon-Nikodym derivative

$$
a_{y}=\frac{\mathrm{d} \tilde{\tau}}{\mathrm{d} \tau}(y)
$$

Proposition A.6 We have

$$
a_{y}=\frac{\mathbf{E}^{\Pi[}\left[r\left(N, \mathcal{A}_{y} N\right) 1(N \in \tilde{\mathbb{M}}) 1\left(\mathcal{A}_{y} N \in \tilde{\mathbb{M}}\right)\right]}{\mathbf{E}^{\Pi}\left[r\left(N, \mathcal{A}_{y} N\right)\right] \Pi(N \in \tilde{\mathbb{M}})} .
$$

Proof: By Lemma A.5

$$
a_{y}=\frac{\mathbf{E}^{\tilde{\Pi}}\left[r\left(N, \mathcal{A}_{y} N\right) 1\left(\mathcal{A}_{y} N \in \tilde{\mathbb{M}}\right)\right]}{\mathbf{E}^{\Pi}\left[r\left(N, \mathcal{A}_{y} N\right)\right]} .
$$

By the truncation property (see Lemma A.3)

$$
\begin{aligned}
\mathbf{E}^{\tilde{\Pi}}\left[r\left(N, \mathcal{A}_{y} N\right) 1\left(\mathcal{A}_{y} N \in \tilde{\mathbb{M}}\right)\right] \\
=\frac{\mathbf{E}^{\Pi}\left[r\left(N, \mathcal{A}_{y} N\right) 1(N \in \tilde{\mathbb{M}}) 1\left(\mathcal{A}_{y} N \in \tilde{\mathbb{M}}\right)\right]}{\Pi(N \in \tilde{\mathbb{M}})},
\end{aligned}
$$

which completes the proof.

We now conclude by showing that that the free process and the loss process of Theorem 4.1 fall within the above framework.

Corollary A.7 If $r\left(\nu, \mathcal{A}_{x} \nu\right) \equiv 1$ and $r\left(\mathcal{A}_{x} \nu, \nu\right)=1 / \tau$ then the free process is reversible and $\Pi$ is the Poisson distribution with mean measure $\tau \lambda$. Its restriction to the set $\tilde{\mathbb{M}}$ of Theorem 4.1 is ergodic. We have the following spatial version of the Erlang formula:

$$
b_{y}=1-a_{y}=\frac{\Pi\left(N \in \tilde{\mathbb{M}}, \mathcal{A}_{y} N \notin \tilde{\mathbb{M}}\right)}{\Pi(N \in \tilde{\mathbb{M}})},
$$

where $b_{y}$ is the rejection or blocking rate.

The fact that the restricted process is is ergodic follows from the fact that it is a SBD process, which satisfies conditions (A.2)-(A.3) with $\mathbb{M}$ replaced by $\tilde{\mathbb{M}}$.

Note that the feasibility probability is the normalizing constant in the spatial Erlang formula. 


\section{References}

[1] F. Baccelli, B. Błaszczyszyn, and M.K. Karray. Up and downlink admission/congestion control and maximal load in large homogeneous CDMA networks. MONET, 9(6):605-617, December 2004 .

[2] F. Baccelli, B. Błaszczyszyn, and F. Tournois. Downlink capacity and admission/congestion control in CDMA networks. In Proc. of IEEE INFOCOM, San Francisco, 2003.

[3] J.S. Evans and D. Everitt. Effective bandwidth-based admission control for multiservice cdma cellular networks. IEEE Transactions on Vehicular Technology, 48, January 1999.

[4] J.S. Evans and D. Everitt. On the teletraffic capacity of cdma cellular networks. IEEE Transactions on Vehicular Technology, 48, January 1999.

[5] K.S. Gilhousen, I.M. Jacobs, R. Padovani, A.J. Viterbi, L.A. Weaver, and C.E. Wheatley. On the capacity of a cellular cdma system. IEEE Trans. Vehic. Technol., pages 303-312, May 1991.

[6] G. Haring, R. Marie, R. Puigjaner, and K. Trivedi. Loss fromulas and their applications to optimization for cellular networks. IEEE trans. on Vehicular Technology, 50, 2001.

[7] X. Huang and R. F. Serfozo. Spatial queueing processes. Math. Oper. Res., 24:865-886, 1999.

[8] M. Karray. "FFactor" — mean formulas for hexagonal CDMA networks with Poisson traffic. private communication, 2003.

[9] F. P. Kelly. Loss networks; special invited paper. Ann. Appl. Probab., 1:319-378, 1991.

[10] J. Laiho, A. Wacker, and T. Novosad. Radio Network Planning and Optimisation for UMTS. Wiley and Sons, 2002.

[11] G. Last and A. Brandt. Marked Point Processes on the Real Line, The Dynamic Approach. Springer, 1995.

[12] Z. Liu and M. El Zarki. Sir-based call admission control for ds-cdma cellular systems. IEEE Sel. Areas in Comm., 12:638 -644, May 1994.

[13] K. Mitchell and K. Sohraby. An analysis of the effects of mobility on bandwidth allocation strategies in multi-class cellular wireless networks. In Proc. of IEEE INFOCOM, 2001.

[14] C. Preston. Spatial birth-and-death processes. Bull. Int. Statist. Inst., 46(2), 1977.

[15] R. Ramjee, R. Nagarajan, and D. Towsley. On optimal call admission control in cellular networks. Wireless Networks, 3:29-41, 1997.

[16] R. Serfozo. Introduction to stochastic networks. Springer, New York, 1999.

[17] R. Serfozo. Reversible markov processes on general spaces: Spatial birth-death and queueing processes. Infomacjonnyje processy, 2(2):259-261, 2002.

[18] M. Sidi and D. Starobinski. New call blocking versus handoff blocking in cellular networks. Wireless Networks, 3, 1997.

[19] D. Stoyan, W. Kendall, and J. Mecke. Stochastic Geometry and Its Applications. John Wiley and Sons, 1987.

[20] A.M. Viterbi and A.J. Viterbi. Erlang capacity of a power controlled CDMA system. IEEE Journal on Selected Areas in Communications, 11(6):892-899, August 1993. 


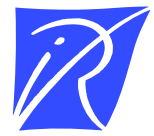

Unité de recherche INRIA Rocquencourt Domaine de Voluceau - Rocquencourt - BP 105 - 78153 Le Chesnay Cedex (France)

Unité de recherche INRIA Lorraine : LORIA, Technopôle de Nancy-Brabois - Campus scientifique 615, rue du Jardin Botanique - BP 101 - 54602 Villers-lès-Nancy Cedex (France)

Unité de recherche INRIA Rennes : IRISA, Campus universitaire de Beaulieu - 35042 Rennes Cedex (France)

Unité de recherche INRIA Rhône-Alpes : 655, avenue de 1'Europe - 38330 Montbonnot-St-Martin (France)

Unité de recherche INRIA Sophia Antipolis : 2004, route des Lucioles - BP 93 - 06902 Sophia Antipolis Cedex (France)

INRIA - Domaine de Voluceau - Rocquencourt, BP 105 - 78153 Le Chesnay Cedex (France)

http://www.inria.fr

ISSN 0249-6399 